\title{
Posterior Arthroscopic Subtalar Arthrodesis: Clinical and Radiologic Review of 19 Cases
}

\author{
Xavier Martín Oliva, MD, PhD ${ }^{1,2}$, Pedro Falcão, $\mathrm{MD}^{3}$, \\ Raul Fernandes Cerqueira, MD ${ }^{4}$, Ricardo Rodrigues-Pinto, MD, PhD, FEBOT ${ }^{5,6}$ \\ ${ }^{1}$ Orthopaedic Surgeon, Department of Orthopaedics, Clinica del Remei, Barcelona, Spain \\ ${ }^{2}$ Professor, Department of Anatomy and Human Embryology, Faculty of Medicine, University of Barcelona, Barcelona, Spain \\ ${ }^{3}$ Orthopaedic Surgeon, Department of Orthopaedics, Centro Hospitalar de Lisboa Central, Hospital Curry Cabral, Lisbon, Portugal \\ ${ }^{4}$ Orthopaedic Surgeon, Department of Orthopaedics, Unidade Local de Saúde do Nordeste, Bragança, Portugal \\ ${ }^{5}$ Orthopaedic Surgeon, Department of Orthopaedic Surgery, Centro Hospitalar do Porto, Hospital de Santo António, Porto, Portugal \\ ${ }^{6}$ Professor, Instituto de Ciências Biomédicas Abel Salazar, University of Porto (ICBAS-UP), Porto, Portugal
}

\section{A R T I C L E I N F O}

Level of Clinical Evidence: 4

Keywords:

ankle arthroscopy

posterior subtalar arthroscopy

subtalar arthritis

subtalar arthrodesis

\begin{abstract}
A B S T R A C T
Arthroscopic subtalar arthrodesis has recently gained popularity in the treatment of primary subtalar or posttraumatic arthritis, coalition, or inflammatory diseases with subtalar arthritis. The present study reports the clinical and radiologic results of 19 patients (19 feet) who underwent posterior arthroscopic subtalar arthrodesis using 2 posterior portals. A total of 19 posterior arthroscopic subtalar arthrodeses (minimum follow-up of 24 months) performed without a bone graft and with 2 parallel screws were prospectively evaluated. The fusion rate was $94 \%$ (mean time to fusion 9.8 weeks). Modified American Orthopaedic Foot and Ankle Society anklehindfoot scale score (maximum 94 points) improved significantly from 43 to 80 points and the visual analog scale for pain score improved from 7.6 to 1.2. The 12-item short-form physical and mental scores at the last follow-up point were 52.5 and 56.4, respectively. One (5.3\%) patient underwent open repeat fusion for nonunion, 2 (10.5\%) patients required a second procedure for implant removal, and 1 (5.3\%) experienced reversible neuropraxia. In conclusion, posterior arthroscopic subtalar arthrodesis is a safe technique with a good union rate and a small number of complications in patients with no or very little hindfoot deformity.
\end{abstract}

(c) 2017 by the American College of Foot and Ankle Surgeons. All rights reserved.
Isolated subtalar arthrodesis is a well-documented and accepted procedure for surgical treatment of subtalar joint pathology presenting with pain, instability, and deformity that does not respond to conservative treatment $(1,2)$. The most frequent indications are primary subtalar or post-traumatic arthritis (secondary to talar or calcaneal fractures), congenital malformations (coalition), or inflammatory diseases (3).

In the past, double (subtalar and talonavicular) or triple arthrodesis (subtalar, talonavicular, and calcaneocuboid) has been the preferred treatment of subtalar arthritis with great deformity. It is known, however, that the function of the talonavicular joint has the greatest influence on the overall hindfoot function. Therefore, isolated subtalar arthrodesis has the advantage of preserving some hindfoot mobility and having a reduced risk of secondary degenerative disease of neighboring joints $(2,4)$.

\footnotetext{
Financial Disclosure: None reported.

Conflict of Interest: None reported.

Address correspondence to: Ricardo Rodrigues-Pinto, MD, PhD, FEBOT, Department of Orthopaedics, Centro Hospitalar do Porto, Hospital de Santo António, Largo Prof. Abel Salazar, Porto 4099-001 Portugal.

E-mail address: ric_pinto@hotmail.com (R. Rodrigues-Pinto).
}

Numerous techniques have been described for the optimal surgical approach, bone graft, method of fixation, and postoperative care (5). Open procedures are most often used but have a greater risk of wound infection, nonunion, and neurovascular injury (2,6). Posterior arthroscopic subtalar arthrodesis (PASTA), performed initially in 1992 and first described by Tasto (7) in 1994, allows for preservation of the blood supply, faster recovery, less pain, preservation of foot proprioception, and fewer complications $(2,6)$. Contraindications to this procedure are previous failed arthrodesis, deformities needing correction, and/or associated procedures that cannot be performed with the patient in the prone position (for posterior portals) (2).

After the advent of arthroscopic subtalar arthrodesis, most surgeons used lateral portals (anterolateral, posterolateral, and accessory) (6). In 2000, van Dijk et al (8) described posterior arthroscopy using posterolateral and posteromedial portals. Since then, these 2 portals (with or without a third portal) have been used for arthroscopic subtalar arthrodesis by several surgeons (6,9-11). Although concerns regarding the safety of these portals have been raised, the thorough understanding of the ankle anatomy and the relationship of the arthroscopic portals to neurovascular structures at risk makes this approach relatively safe (12). 
The objective of the present report was to describe the operative technique and clinical and radiologic results of 19 cases of PASTA using a 2-posterior portal approach with the patient in the prone position.

\section{Patients and Methods}

From January 2008 to September 2012, 19 subtalar arthrodeses were performed in 19 patients in the same Institution by the same surgeon. The data were prospectively collected. The data from all 19 patients were available for retrospective analysis. All participants in the present study provided informed consent.

All patients had isolated subtalar arthritis. The exclusion criteria for arthroscopic arthrodesis were a hindfoot coronal deformity (varus of $>5^{\circ}$ or valgus of $>15^{\circ}$ ), previous or active infection, previous failed arthrodesis, and the need for a concomitant procedure. The main indication for surgery was post-traumatic subtalar arthritis ( 8 patients), followed by primary arthritis ( 7 patients), inflammatory disease (rheumatoid arthritis in 2 and psoriatic arthritis in 1), and tarsal coalition (1 patient). Of the 19 patients, 12 were male and 7 were female. Their mean age at surgery was 50.9 (range 35 to 69) years. The mean follow-up period was 42.9 (range 24 to 68) months.

The patients were clinically evaluated at 2, 6, 8 to 9, 12, and 24 weeks and annually thereafter. Radiographs were taken at all but the first (2-week) appointment. The patients were clinically evaluated preoperatively and at the last follow-up examination using the visual analog scale (VAS) for pain and the modified ankle-hindfoot American Orthopaedic Foot and Ankle Society (AOFAS) score, in which the scoring system was modified to eliminate points from subtalar motion (maximum possible score of 94 points), as previously described (13). The 12-item short-form questionnaire (SF-12) was used to record the patient-reported outcomes to assess the patients' physical and mental status at the last follow-up examination.

Union was determined at the monthly appointments by clinical (no pain and no subtalar motion) and radiographic (x-ray and/or computed tomography) evaluation. When trabecular bridging at the posterior facet was not seen at the 8- to 9-week appointment, a computed tomography scan was ordered to evaluate for fusion $(n=13)$. The complications present at the last follow-up visit were also recorded.

Statistical analysis was performed using the software InStat Prism, version 6.0 (GraphPad, La Jolla, CA). The Shapiro-Wilk test was used to confirm a normal distribution between samples, and a paired Student $t$ test was used to compare the preoperative and postoperative AOFAS and VAS scores. A $p$ value $<.05$ was considered to represent a statistically significant difference.

\section{Operative Technique}

All procedures were performed with the patient under general or regional anesthesia. A tourniquet was used, and prophylactic antibiotics were administered. The patients were placed prone with the foot outside the operating table edge. A 2-portal posterior arthroscopy was performed, as previously described (8). No traction and no other portals were used. First, the posterolateral portal was created $1 \mathrm{~cm}$ proximal to the tip of lateral malleolus just lateral to Achilles tendon, after which a $4.5-\mathrm{mm} 30^{\circ}$ optic arthroscope was inserted. Next, at the same level and immediately medial to the Achilles tendon, a posteromedial portal was created. A 4.0-mm shaver was inserted and angled laterally to touch arthroscope shaft. This angulation avoids injury to the neurovascular structures that would occur if the shaver was introduced in a straight line. Debridement and synovectomy were then performed, taking care to identify and preserve the flexor hallucis longus tendon, because the neurovascular bundle is just medial to the tendon.
Next, the posterior joint capsule was removed to perfectly identify the subtalar joint. Then, decortication with removal of cartilage and approximately $2 \mathrm{~mm}$ of the subchondral bone was performed using curettes, chisels, and burrs (Fig. 1). Only the posterior facet was prepared, with the anterior limit the interosseous ligament. Once a normal bone contour was achieved, fixation was performed with 2 percutaneous 6.5$\mathrm{mm}$ screws introduced from the calcaneus to the talus (posteriorly and plantarly to anteriorly and dorsally). The screws were inserted in a parallel configuration under fluoroscopic guidance. No bone graft or bone substitute was used in any case. The skin incisions were sutured, and a plaster boot was applied.

Postoperatively, a non-weightbearing plaster boot was used for 4 weeks and was then changed to a walker boot with partial weightbearing for 2 weeks. At 6 weeks, the patients were allowed to start full weightbearing, using the walker boot until union was achieved. The patients were clinically and radiographically followed up for a minimum period of 24 months.

\section{Results}

The Table presents the patient demographics and clinical and radiographic analysis. All but 1 (5.3\%) patient achieved subtalar fusion at a mean \pm standard deviation of $9.8 \pm 1.1$ weeks, indicating an incidence of fusion of $94.7 \%$. The patient with nonunion underwent open arthrodesis with a bone graft at 15 months after the index surgery. Therefore, data analysis was performed for the remaining 18 patients.

The modified AOFAS score (maximum 94 points) had significantly improved in all 18 (94.7\%) patients in whom fusion was achieved from a mean preoperative score of $42.9 \pm 9.1$ to a mean postoperative score of $80.2 \pm 4.8(p<.001)$. Two $(10.5 \%)$ patients did not complete the VAS score. For the remaining $16(84.2 \%)$ patients, it improved from a mean preoperative score of $7.6 \pm 1.0$ to a score of $1.2 \pm 1.2$ at the last followup visit $(p<.001)$. At the last follow-up visit, the SF-12 physical composite score was $52.5 \pm 6.7$ and the mental composite score was $56.4 \pm 7.4$. Fig. 2 shows typical radiographic and computed tomography images obtained 10 months after surgery.

Complications occurred in 4 (21.1\%) of 19 patients. Of the 4 patients, 1 (5.3\%) developed nonunion, which was revised using an open approach; 2 (10.5\%) required implant removal at 7 and 9 months; and $1(5.3 \%)$ patient developed hypoesthesia in the plantar side of the foot that had resolved spontaneously 6 months after surgery. No other complications, such as permanent nerve injury, infection, malalignment, or complex regional pain syndrome, were observed.

\section{Discussion}

Since van Dijk et al (8) described the technique for ankle arthroscopy using 2 posterior portals, foot and ankle surgeons have had increasing interest in PASTA. Although technically more demanding than other ankle arthroscopic procedures, subtalar arthroscopy offers a less-invasive approach with many potential

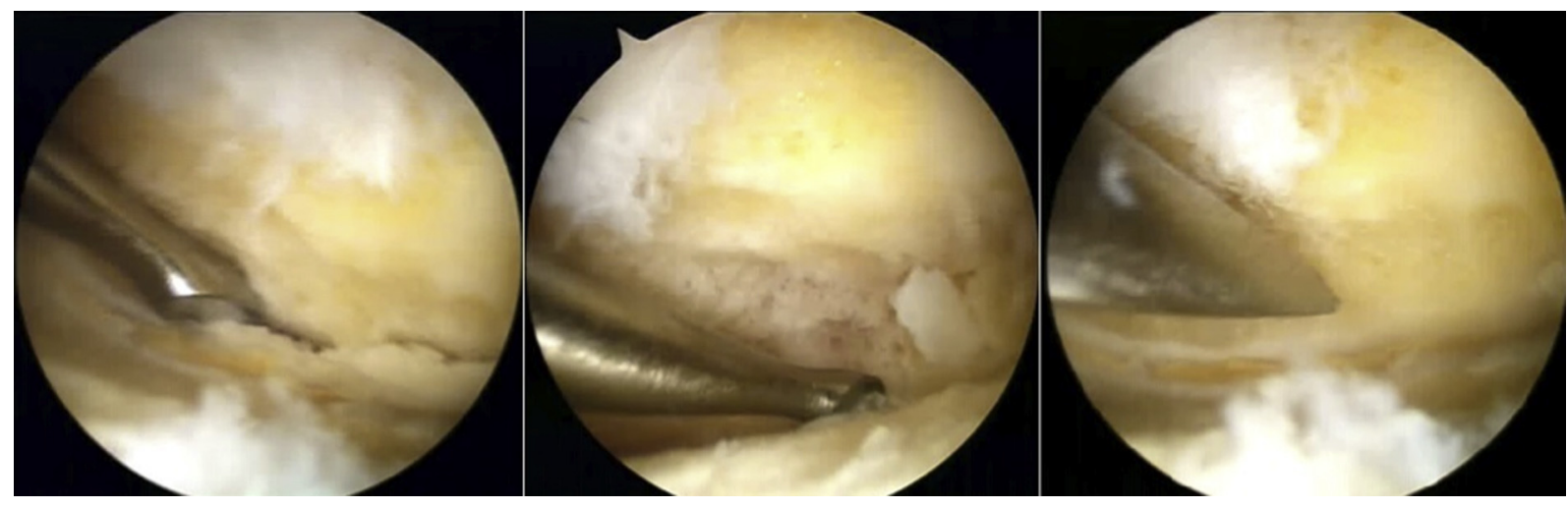

Fig. 1. Posterior facet decortications with curette and chisel. 
Table

Patient demographics and results

\begin{tabular}{|c|c|c|c|c|c|c|c|c|c|c|c|c|}
\hline \multirow[t]{2}{*}{ Pt. No. } & \multirow[t]{2}{*}{ Gender } & \multirow[t]{2}{*}{ Age (yr) } & \multirow[t]{2}{*}{ Indication for surgery } & \multirow[t]{2}{*}{ Follow-Up (mo) } & \multicolumn{2}{|c|}{ AOFAS Score } & \multicolumn{2}{|c|}{ VAS Score } & \multicolumn{2}{|c|}{ SF-12 Score } & \multirow[t]{2}{*}{ Time to Fusion (wk) } & \multirow[t]{2}{*}{ Complications } \\
\hline & & & & & Pre & Post & Pre & Post & PCS & MCS & & \\
\hline$\overline{1}$ & Female & 45 & Tarsal coalition & 66 & 33 & 74 & 8 & 2 & 51.5 & 61.6 & 12 & None \\
\hline 2 & Female & 39 & Inflammatory & 68 & 56 & 84 & 7 & 0 & 56.7 & 60.8 & 9 & None \\
\hline 3 & Female & 43 & Inflammatory & 24 & 39 & 82 & 8 & 1 & 53.8 & 55 & 8 & None \\
\hline 4 & Male & 41 & Primary & 48 & 53 & 71 & NA & NA & 43.8 & 62.1 & 9 & None \\
\hline 5 & Male & 49 & Traumatic & 65 & 39 & 78 & 9 & 3 & 61.4 & 37.3 & 10 & None \\
\hline 6 & Male & 48 & Primary & 35 & 33 & 78 & 7 & 1 & 56.2 & 59.8 & 9 & Painful hardware \\
\hline 7 & Male & 67 & Traumatic & 27 & 39 & 82 & 8 & 1 & 53.8 & 57.9 & 11 & None \\
\hline 8 & Female & 58 & Primary & 60 & 45 & 84 & 7 & 0 & 56.6 & 60.8 & 11 & None \\
\hline 9 & Male & 59 & Traumatic & 62 & 45 & 85 & 8 & 2 & 54.8 & 57.1 & 10 & None \\
\hline 10 & Male & 52 & Primary & 56 & 59 & 78 & 7 & 2 & 56.2 & 59.8 & 11 & None \\
\hline 11 & Female & 35 & Traumatic & 46 & 53 & 84 & 6 & 1 & 41.4 & 59.2 & 9 & None \\
\hline 12 & Male & 56 & Primary & 43 & 39 & 85 & NA & NA & 55.3 & 60.7 & 11 & None \\
\hline 13 & Male & 69 & Traumatic & 24 & 33 & 70 & 9 & 4 & 33.9 & 52.5 & 9 & Neuropraxia \\
\hline 14 & Male & 46 & Traumatic & 34 & 33 & 78 & 8 & 1 & 50 & 58.8 & 9 & Painful hardware \\
\hline 15 & Female & 47 & Inflammatory & 36 & 56 & 84 & 6 & 0 & 55.9 & 58.7 & 10 & None \\
\hline $16^{*}$ & Male & 54 & Traumatic & 27 & 33 & NA & 8 & NA & NA & NA & NA & Nonunion \\
\hline 17 & Male & 45 & Primary & 31 & 39 & 85 & 9 & 0 & 56.8 & 57.9 & 11 & None \\
\hline 18 & Female & 61 & Traumatic & 29 & 33 & 78 & 8 & 1 & 50.7 & 37.2 & 9 & None \\
\hline 19 & Male & 54 & Primary & 35 & 45 & 84 & 7 & 0 & 55.9 & 58.7 & 9 & None \\
\hline Mean & NA & 50.9 & NA & 42.9 & 42.4 & 80.2 & 7.6 & 1.2 & 52.5 & 56.4 & 9.8 & None \\
\hline SD & NA & 9.2 & NA & 15.5 & 9.1 & 4.8 & 1.0 & 1.2 & 6.7 & 7.4 & 1.1 & None \\
\hline
\end{tabular}

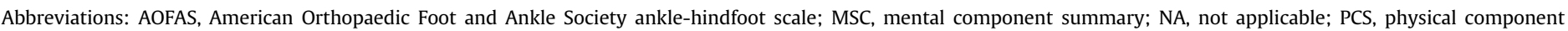
summary; Post, postoperative; Pre, preoperative; Pt. No., patient number; SD, standard deviation; SF-12, 12-item short-form questionnaire; VAS, visual analog scale.

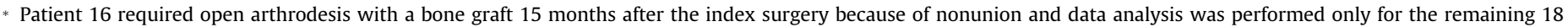
patients.

advantages compared with the traditional open methods (14). These include preservation of the blood supply, faster recovery, less pain, preservation of foot proprioception, and fewer complications $(2,6)$. However, if a significant hindfoot deformity exists, subtalar arthrodesis should not be addressed using an arthroscopic procedure.

Several investigators have used this technique and reported high union rates and few complications. Amendola et al (9) reported on a series of 11 patients who underwent PASTA using bone graft, with only 1 nonunion and a mean fusion time of 10 weeks. The AOFAS score improved from 36 to 86 (9). Carro et al (15) and Beimers et al (10) reported a 100\% fusion rate in 2 small series of 4 and 3 patients, respectively. El Shazly et al (16) also reported a $100 \%$ fusion rate in 10 patients after a mean interval of 11 weeks and an improvement in the AOFAS score from 38 to 74. More recently, Lee et al (6) reported on a series of 16 patients, with a $94 \%$ fusion rate after 11 weeks. In a series of 10 patients, using a bone graft, Albert et al (14) reported a 100\% fusion rate and a mean fusion time of only 7 weeks.

All these studies showed an improvement in the interval to union and the union rate compared with open procedures. Easley et al (3) performed a review of a large series of 148 isolated open subtalar arthrodesis procedures and found a fusion rate of $84 \%$ after primary arthrodesis at an average time of 10 to 15 weeks postoperatively.

When ankle arthroscopy using posterolateral and posteromedial portals was initially described, concerns were raised about the safety of this procedure. However, an anatomic study investigating the relationships between the portals and neurovascular structures found that the sural nerves are a relatively safe distance $(9.7 \mathrm{~mm})$ from the posterolateral portal (12). The structures closer to the posteromedial portal were the posterior tibial nerve and artery, at $7.27 \mathrm{~mm}$ and $11.46 \mathrm{~mm}$, respectively, from that portal (12). Sitler et al (17) also analyzed the distance to the medial calcaneal nerve, which was, on average, $17.1 \mathrm{~mm}$ from the posteromedial portal. However, because the patient is prone, the neurovascular structures will drift anteriorly, reducing the risk of injury (6). These results demonstrate that when the portals are placed close to the Achilles tendon, the instruments are introduced in the correct direction (pointing toward the midline middle), and, using the flexor hallucis longus as a medial boundary marker, the posterior portals will be safe to use $(6,12,17)$.
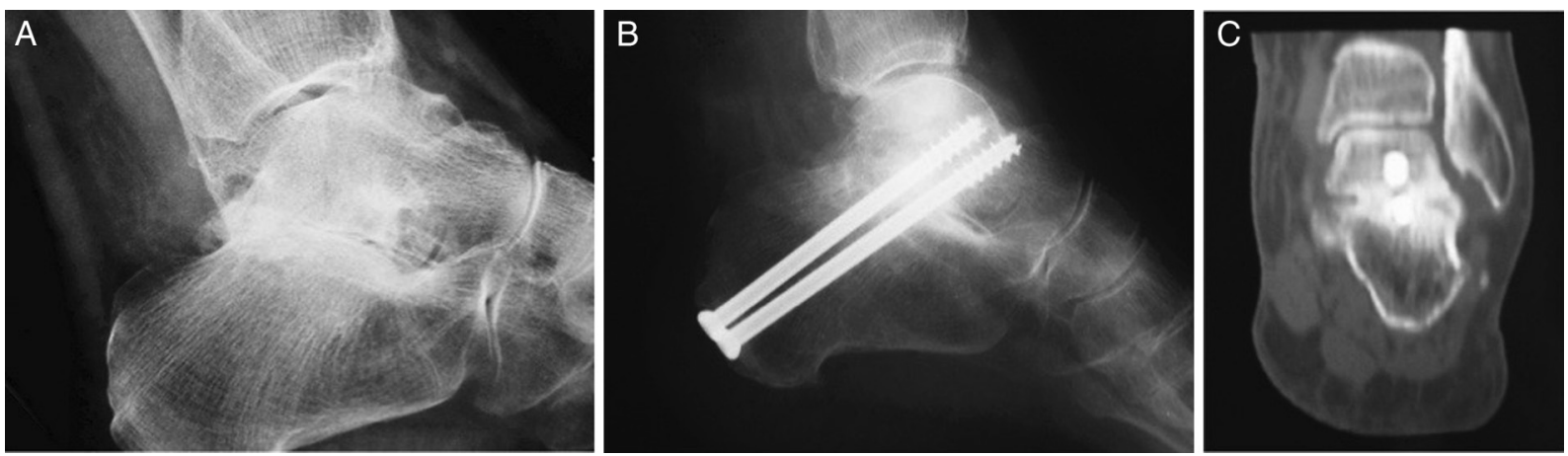

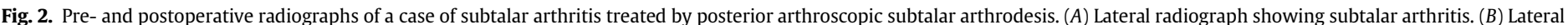

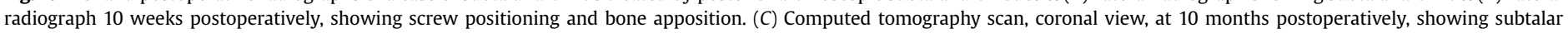
fusion with 2 screws. 
One controversial aspect of this approach is related to the number and orientation of the fixation screws. DeCarbo et al (18) compared the use of a single- versus a 2-screw construct and found no differences in the union rate. However, different 2-screw fixation techniques have been reported. In a cadaver study, Chuckpaiwong et al (19) compared single-screw, double-parallel screw, and doubledivergent screw configurations and found that double-divergent screws conferred the greatest compression, greatest torsional stiffness, and the least amount of joint rotation. However, controlled clinical trials are necessary to substantiate this finding.

To the best of our knowledge, the present study is the largest series of patients with longer follow-up who underwent PASTA. The AOFAS modified ankle-hindfoot scale score was used for clinical assessment, together with the VAS score. The AOFAS score, although not fully validated, has been widely used in the field, and a recent study proposed it as the ideal score for clinical assessment of foot and ankle pathology, as it has been used in $55.9 \%$ of studies (20). It should be noted that the subjective component of the AOFAS score has been shown to produce valid information (21). A significant improvement from 42.4 to 80.2 of 94 points was seen, with improvement in the VAS score from 7.6 to 1.4 , demonstrating a substantive decrease in pain intensity after arthroscopic fusion in this cohort of patients.

Patient-reported outcome measures are being increasingly used to assess patient outcomes after medical and surgical procedures (22), and SF-12 has been widely studied to assess the clinical results after foot and ankle procedures (23-25). In the present study, the SF-12 physical and mental component scores at the last follow-up visit were 52.5 and 56.4, respectively. No major complications were found. The fusion rate was $94 \%$ at 9.9 weeks, without a bone graft, and with a 2-parallel screw configuration used for fixation.

In conclusion, the results of the present study add strength to previous early reports of PASTA but with a larger cohort of patients and longer follow-up period. We found PASTA to be a reliable and safe technique to achieve fusion with a small complication rate in patients with no or very little hindfoot deformity.

\section{References}

1. Boffeli TJ, Reinking RR. A 2-screw fixation technique for subtalar joint fusion: a retrospective case series introducing a novel 2-screw fixation construct with operative pearls. J Foot Ankle Surg 51:734-738, 2012.

2. Muraro GM, Carvajal PF. Arthroscopic arthrodesis of subtalar joint. Foot Ankle Clin 16:83-90, 2011.
3. Easley ME, Trnka HJ, Schon LC, Myerson MS. Isolated subtalar arthrodesis. J Bone Joint Surg Am 82:613-624, 2000.

4. Savory KM, Wulker N, Stukenborg C, Alfke D. Biomechanics of the hindfoot joints in response to degenerative hindfoot arthrodeses. Clin Biomech (Bristol, Avon) 13:62-70, 1998.

5. Tuijthof GJ, Beimers L, Kerkhoffs GM, Dankelman J, Dijk CN. Overview of subtalar arthrodesis techniques: options, pitfalls and solutions. Foot Ankle Surg 16:107116, 2010.

6. Lee KB, Park CH, Seon JK, Kim MS. Arthroscopic subtalar arthrodesis using a posterior 2-portal approach in the prone position. Arthroscopy 26:230-238, 2010.

7. Tasto J. Subtalar Arthroscopy. Operative Arthroscopy, ed 3, Lippincott Williams \& Wilkins, New York, 2002, pp. 944-952.

8. van Dijk CN, Scholten PE, Krips R. A 2-portal endoscopic approach for diagnosis and treatment of posterior ankle pathology. Arthroscopy 16:871-876, 2000.

9. Amendola A, Lee KB, Saltzman CL, Suh JS. Technique and early experience with posterior arthroscopic subtalar arthrodesis. Foot Ankle Int 28:298-302, 2007.

10. Beimers L, de Leeuw PA, van Dijk CN. A 3-portal approach for arthroscopic subtalar arthrodesis. Knee Surg Sports Traumatol Arthrosc 17:830-834, 2009.

11. Gómez J, Musatadi M, Martínez J. Artrodesis subastragalina artroscópica. Rev Esp Cir Osteoart 45:1-4, 2010.

12. Martin Oliva X, Mendez Lopez JM, Monzo Planella M, Bravo A, Rodrigues-Pinto R. Anatomical relations of anterior and posterior ankle arthroscopy portals: a cadaveric study. Eur J Orthop Surg Traumatol 25:577-581, 2014.

13. Lundeen RO. Arthroscopic fusion of the ankle and subtalar joint. Clin Podiatr Med Surg 11:395-406, 1994.

14. Albert A, Deleu PA, Leemrijse T, Maldague P, Devos Bevernage B. Posterior arthroscopic subtalar arthrodesis: ten cases at one-year follow-up. Orthop Traumatol Surg Res 97:401-405, 2011.

15. Carro LP, Golano P, Vega J. Arthroscopic subtalar arthrodesis: the posterior approach in the prone position. Arthroscopy 23:445.e1-445.e4, 2007.

16. El Shazly O, Nassar W, El Badrawy A. Arthroscopic subtalar fusion for posttraumatic subtalar arthritis. Arthroscopy 25:783-787, 2009.

17. Sitler DF, Amendola A, Bailey CS, Thain LM, Spouge A. Posterior ankle arthroscopy: an anatomic study. J Bone Joint Surg Am 84-A:763-769, 2002.

18. DeCarbo WT, Berlet GC, Hyer CF, Smith WB. Single-screw fixation for subtalar joint fusion does not increase nonunion rate. Foot Ankle Spec 3:164-166, 2010.

19. Chuckpaiwong B, Easley ME, Glisson RR. Screw placement in subtalar arthrodesis: a biomechanical study. Foot Ankle Int 30:133-141, 2009.

20. Hunt KJ, Hurwit D. Use of patient-reported outcome measures in foot and ankle research. J Bone Joint Surg Am 95, 2013. e118(1-9).

21. Ibrahim T, Beiri A, Azzabi M, Best AJ, Taylor GJ, Menon DK. Reliability and validity of the subjective component of the American Orthopaedic Foot and Ankle Society clinical rating scales. J Foot Ankle Surg 46:65-74, 2007.

22. Black N. Patient reported outcome measures could help transform healthcare. BM] 346:f167, 2013.

23. Ajis A, Tan KJ, Myerson MS. Ankle arthrodesis vs TTC arthrodesis: patient outcomes, satisfaction, and return to activity. Foot Ankle Int 34:657-665, 2013.

24. Coughlin MJ, Grimes JS, Traughber PD, Jones CP. Comparison of radiographs and CT scans in the prospective evaluation of the fusion of hindfoot arthrodesis. Foot Ankle Int 27:780-787, 2006.

25. Ellington JK, Gupta S, Myerson MS. Management of failures of total ankle replacement with the agility total ankle arthroplasty. J Bone Joint Surg Am 95:2112-2118, 2013. 